\title{
Signo de la nariz de payaso como manifestación de neoplasia pulmonar
}

\section{Clown nose sign as a manifestation of pulmonary neoplasia}

\author{
Ricardo F. Rojas-López', Jorge L. Bermúdez-Flórez², Diana I. Conde-Hurtado ${ }^{3 *}$, \\ Óscar F. Valdivieso-Cárdenas ${ }^{4}$, Nathalia Garavito-Hernández ${ }^{5}$ e Isabel C. Bolívar-Aguilera ${ }^{5}$
}

${ }^{1}$ Servicio de Dermatología, Clínica FOSCAL Internacional; ${ }^{2}$ Servicio de Dermatología, Universidad Autónoma de Bucaramanga (UNAB); ${ }^{3}$ Servicio de Medicina, Universidad Autónoma de Bucaramanga (UNAB); ${ }^{4}$ Servicio de Radiología, Radiólogos Especializados; ${ }^{5}$ Servicio de Patología, Isabel Bolivar Laboratorio. Floridablanca, Colombia

\section{Resumen}

Introducción: Las metástasis cutáneas son diseminaciones de células malignas en la piel de tumores distantes que deben sospecharse en lesiones de rápido crecimiento sin respuesta al tratamiento. Representan un reto clínico pues no tienen una morfología macroscópica especifica, mientras que histológicamente se localizan en dermis e hipodermis (carecen de componente in situ). Presentación del caso: Se describe el caso de una mujer adulta mayor quien presenta metástasis en nariz de payaso tras desarrollar cáncer de pulmón con dos variantes distintas: adenocarcinoma y carcinoma escamocelular mal diferenciado macrocítico. Se confirmó la relación con estudio de inmunohistoquímica positivo para marcadores de carcinoma escamocelular pobremente diferenciado. Discusión: El signo de la nariz de payaso es una lesión inflamatoria nodular en la piel de la punta nasal como manifestación de metástasis cutánea de un tumor visceral. Se ha identificado junto con múltiples neoplasias entre las que se destacan el cáncer de pulmón en hombres y el cáncer mamario en mujeres. A pesar de su baja frecuencia se debe tener en cuenta por su efecto en la sobrevida de los pacientes. Conclusiones: La aparición de lesiones inflamatorias en punta nasal de rápido crecimiento que no respondan al tratamiento obliga realizar estudios de extensión para analizar la etiología.

Palabras clave: Metástasis de la neoplasia. Neoplasias pulmonares. Neoplasias cutáneas. Neoplasias nasales. Nariz. Inmunohistoquímica

\section{Abstract}

Introduction: Cutaneous metastases are sowings of malignant cells in the skin of distant tumors that should be suspected in rapidly growing lesions without response to treatment. They represent a clinical challenge because they do not have a specific macroscopic morphology, while histologically they are located in the dermis and hypodermis (they lack an in situ component). Case presentation: We describe the case of an older adult woman who has metastasis in Clown Nose after developing lung cancer with two different variants: adenocarcinoma and squamous cell carcinoma poorly differentiated from large cells. In whom the association with positive immunohistochemical study for markers of poorly differentiated squamous

\section{Correspondencia:}

*Diana I. Conde-Hurtado

E-mail: dconde@unab.edu.co
Fecha de recepción: 29-05-2020

Fecha de aceptación: 16-08-2020

DOI: 10.24875/MCUT.20000022
Disponible en internet: 13-05-2021 Med Cutan Iber Lat Am. 2021;49(1):25-29

www.MedicinaCutanealLA.com 0210-5187/@ 2020 Colegio Ibero Latinoamericano de Dermatología A.C. (CILAD). Publicado por Permanyer. Este es un artículo open access bajo la licencia CC BY-NC-ND (http://creativecommons.org/licenses/by-nc-nd/4.0/). 
cell carcinoma was confirmed. Discussion: The Clown Nose Sign is a nodular inflammatory lesion in the skin of the nasal tip as a manifestation of cutaneous metastasis of a visceral tumor. It has been associated with multiple neoplasms specially lung cancer in men and breast cancer in women. Despite its low frequency, it must be taken into account due to its impact on patient survival. Conclusions: The appearance of inflammatory lesions in nasal tip of rapid growth that do not respond to therapy, requires extension studies to analyze its etiology.

Key words: Neoplasm metastasis. Lung neoplasms. Skin neoplasms. Nose neoplasms. Nose. Immunohistochemistry

\section{Introducción}

El signo de la nariz de payaso es un tipo de metástasis cutánea caracterizada por la presencia de una lesión nodular o placa eritematosa violácea que afecta la punta nasal y se asemeja a la nariz roja de un payaso ${ }^{1}$. Desde su primera descripción en $1990^{2}$ se ha relacionado con múltiples neoplasias malignas, las más relevantes de las cuales son el cáncer de pulmón en hombres y el cáncer de mama en mujeres ${ }^{3}$. A pesar de que las metástasis cutáneas se presentan en sólo $0.7 \%$ a $9 \%$ de todos los pacientes con cáncer, es un diagnóstico que debe identificarse en fase temprana por sus efectos en la sobrevida de los pacientes, ya que indica enfermedad diseminada y de mal pronóstico, con una sobrevida promedio de cinco meses ${ }^{4,5}$.

Se describe el caso de una mujer adulta mayor con este signo con el objetivo de resaltar la posibilidad diagnóstica de metástasis cutánea en lesiones de piel de la punta nasal de rápido crecimiento que no responden al tratamiento convencional.

\section{Caso clínico}

Se presenta el caso de una paciente femenina de 71 años, quien asiste al servicio de dermatología por un cuadro clínico de tres meses de crecimiento progresivo de un nódulo en la punta nasal. Al examen físico, paciente Fototipo Fitzpatrick III, con nódulo ertitematovioláceo que deforma la punta nasal de $40 \times 50 \mathrm{~mm}$ de área (Fig. 1), doloroso a la palpación, de bordes mal definidos, superficie lisa y acentuación folicular, piel perilesional con dermatoheliosis.

Al revisar los antecedentes, la paciente refiere encontrarse en estudio de masa pulmonar por lo que aporta radiografía de tórax (Fig. 2) que revela una lesión de $52 \times 67 \mathrm{~mm}$ en el lóbulo superior izquierdo, en contacto estrecho con el mediastino.

Con base en los hallazgos clínicos y paraclínicos, hasta ese momento, se indicaron estudios de extensión para la lesión cutánea con impresión diagnóstica de neoplasia secundaria de piel.

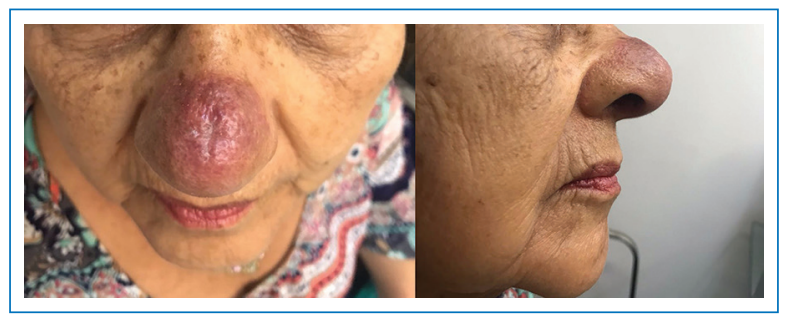

Figura 1. Paciente con signo de la nariz de payaso, vista frontal y lateral.

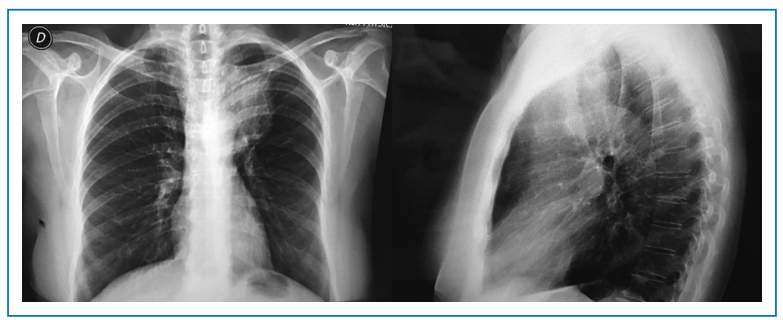

Figura 2. Radiografía de tórax anteroposterior y lateral.

La ecografía de los tejidos blandos de la punta nasal describe una lesión infiltrante de aspecto sólido isoecógeno de bordes irregulares, sin calcificaciones, que se localiza entre los dos cartílagos alares. La valoración con Doppler muestra vascularidad central y periférica. Se establece por medios ecográficos una lesión tumoral sólida en la punta nasal indicativa de localización secundaria.

El servicio de dermatología realizó biopsia incisional de piel de la punta nasal que reveló un tumor maligno escasamente diferenciado en dermis profunda, sin componente in situ en epidermis (Fig. 3).

El estudio de inmunohistoquímica revela un carcinoma escamocelular pobremente diferenciado (Fig. 4) (Tabla 1). Con posterioridad se tomaron muestras del pulmón izquierdo por toracoscopia en la que se reconocen dos tumores de diferentes características. El primero se halla en el lóbulo inferior y corresponde a un adenocarcinoma infiltrante, con compromiso de la pleura visceral; el segundo se localiza en el lóbulo superior y representa un carcinoma escamocelular mal diferenciado macrocítico.

Al considerar la lesión tumoral de rápido crecimiento en la cara y los hallazgos notificados en las herramientas 


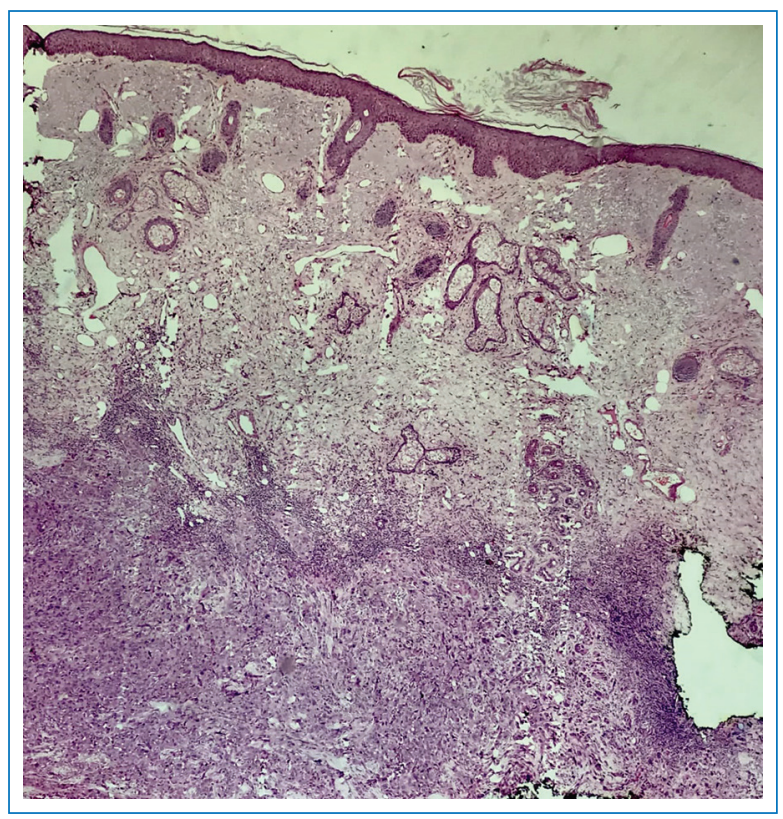

Figura 3. Estudio de histopatologia de punta nasal (Hematoxilina-Eosina, 4×).

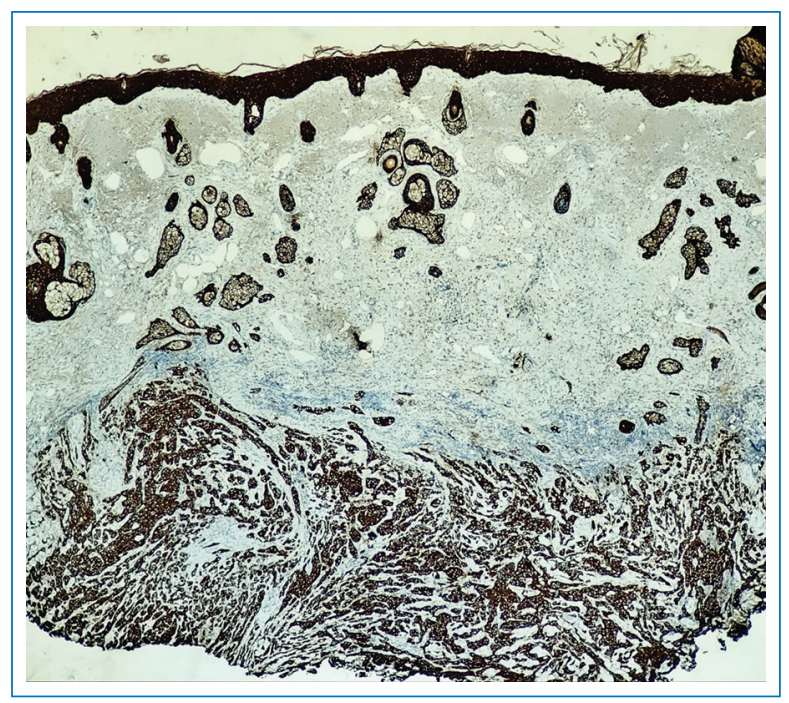

Figura 4. Positividad para cóctel de citoqueratinas en muestra de punta nasal.

diagnósticas se determina metástasis cutánea de origen pulmonar, con características del signo de la nariz de payaso; se le explica a la paciente y se remite a oncología clínica su tratamiento.

\section{Comentario}

Las metástasis cutáneas son diseminaciones de células malignas en la piel procedentes de tumores a
Tabla 1. Perfil inmunohistoquímico de la muestra de la punta nasal

\begin{tabular}{|l|l|}
\hline Marcador & Reactividad \\
\hline Coctel de citoqueratinas & Positivo \\
\hline Citoqueratina 7 & \\
\hline P40 & \\
\hline Vimentina & Negativo \\
\hline Antígeno carcinoembrionario (CEA) & \\
\hline Calretinina & \\
\hline Cromogranina & \\
\hline TTF 1 & \\
\hline Napsina A & \\
\hline Citoqueratina 20 & \\
\hline Melan A & \\
\hline S100 & \\
\hline Receptores de estrógenos & \\
\hline WT1 & \\
\hline Proteína de líquido quístico mamario (GCDFP-15) & \\
\hline
\end{tabular}

distancia ${ }^{6}$. Se consideran manifestaciones infrecuentes de neoplasias porque se presentan en el $0.7 \%$ a $9 \%$ de todos los pacientes con cáncer y deben sospecharse en pacientes con lesiones de rápido crecimiento sin respuesta al tratamiento médico ${ }^{7}$. Se calcula que aparecen uno a tres años después del diagnóstico del tumor primario ${ }^{8}$.

Pueden corresponder a una recaída de una tumoración en remisión (75\%), primera manifestación de una neoplasia de órgano interno (19\%) o diseminación de un tumor primario ya conocido $(7.6 \%)^{8-10}$.

Aunque no tienen una morfología específica, por lo regular se manifiestan en la forma de nódulos, placas o pápulas de rápido crecimiento, redondeados u ovalados y con una coloración rojiza o violácea. Estas lesiones pueden ser dolorosas, ocasionar prurito, ulcerarse, sangrar con facilidad 0 , por el contrario, ser del todo asintomáticas ${ }^{5,11,12}$.

Los sitios más frecuentes son el tórax y abdomen, seguidos de la cara y el cuero cabelludo, mientras que las extremidades son la localización más rara ${ }^{4}$. Los tumores que más emiten metástasis a piel varían de acuerdo con el género. En los hombres, el principal origen es el cáncer de pulmón, seguido por colon y recto, riñón y tracto gastrointestinal (no colorrectal). En 
Tabla 2. Tumores primarios relacionados con metástasis en la nariz de payaso

\begin{tabular}{|l|l|l|}
\hline Cáncer de pulmón & Cáncer de mama & Cáncer esofágico \\
\hline Cáncer de cérvix & Hepatocarcinoma & Leucemia \\
\hline Seminoma & Tumores renales & Cordoma \\
\hline Cáncer de tiroides & & \\
\hline
\end{tabular}

mujeres, la mayor parte proviene del cáncer de mama, seguido por los cánceres colorrectal, pulmonar, ovárico y cervical ${ }^{13}$.

El cáncer de pulmón es el tumor que más rápido proyecta metástasis a piel (5.7 meses después del diagnóstico inicial) y el tumor indiferenciado es la variante histopatológica la que más metastiza a piel $^{4,9,10,12}$.

En el plano histopatológico, las metástasis cutáneas se diferencian de los carcinomas primarios por la ausencia del componente in situ y la localización profunda (dermis e hipodermis) ${ }^{14}$. En los casos en los que esta distinción es difícil, las tinciones de inmunohistoquímica pueden ayudar a dilucidar el diagnóstico ${ }^{15}$. Las características microscópicas de metástasis se asemejan a las del tumor primario, con menor grado de diferenciación ${ }^{11,16}$.

En las ultrasonografías se observan como nódulos subcutáneos, hipoecoicos, homogéneos y bien definidos, y el Doppler a color muestra gran vascularización ${ }^{17}$. El signo de la nariz de payaso es un término que se refiere a una lesión inflamatoria nodular de coloración rojiza o violácea en la punta nasal, secundaria a una metástasis de un tumor visceral. Es extremadamente rara y su presencia debe alertar sobre la posibilidad de neoplasias malignas, metástasis de un tumor visceral, óseo o leucemia (Tabla 2) $)^{1-3}$.

No se conoce con certeza el mecanismo fisiopatológico por el cual ocurre este fenómeno ${ }^{18}$, pero Baston sugirió en 1940 la migración de células tumorales a través de vasos sanguíneos y linfáticos pulmonares ${ }^{19}$. Otros mecanismos son el embolismo de células tumorales a través de plexos venosos hasta los senos venosos del cráneo por elevación de la presión intratorácica y de allí la extensión a la punta nasal ${ }^{18}$. Nuevos informes sugieren implantación yatrogénica de células tumorales cuando existe comunicación entre el tumor primario con la nariz (uso crónico de sonda nasogástrica) ${ }^{20}$.

El signo de la nariz de payaso puede tener una amplia variedad de diagnósticos diferenciales, entre ellos rinofima, rinoescleroma, lupus pernio, lupus túmido, seudolinfoma, granuloma facial, carcinoma escamocelular primario de piel, carcinoma basocelular, entre otros ${ }^{1,3,21}$.

Las metástasis cutáneas se subdiagnostican con frecuencia o se confunden con otras dermatosis porque los hallazgos suelen ser sutiles e inespecíficos ${ }^{5}$. Se requiere un tratamiento interdisciplinario ${ }^{1}$ para conservar la calidad de vida del paciente.

\section{Conclusión}

La aparición de lesiones inflamatorias en la punta nasal de rápido crecimiento que no responden al tratamiento convencional obliga solicitar estudios de extensión para descartar metástasis cutáneas de neoplasias internas, en especial de pulmón y mama.

\section{Conflicto de intereses}

Los autores declaran no tener ningún conflicto de intereses.

\section{Financiamiento}

Los autores declaran que esta investigación no ha recibido ninguna beca específica de agencias de los sectores público, comercial o sin ánimo de lucro.

\section{Responsabilidades éticas}

Protección de personas y animales. Los autores declaran que para esta investigación no se han realizado experimentos en seres humanos ni en animales.

Confidencialidad de los datos. Los autores declaran que han seguido los protocolos de su centro de trabajo sobre la publicación de datos de pacientes.

Derecho a la privacidad y consentimiento informado. Los autores han obtenido el consentimiento informado de los pacientes y/o sujetos referidos en el artículo. Este documento obra en poder del autor de correspondencia.

\section{Bibliografía}

\footnotetext{
1. Colletti G, Allevi F, Moneghini L, Palvarini M. Clown nose: a case of disfiguring nodular squamous cell carcinoma of the face. BMJ Case Rep. 2014;bcr2013200471.

2. Soyer HP, Cerroni L, Smolle J, Kerl H. "Clown nose" skin metastasis of breast cancer. Z Hautkr. 1990;65:929-31.

3. Shindo M, Yoshida Y, Tominaga K, Yamamoto O. Skin metastasis of hypopharyngeal carcinoma to the nasal tip. Yonago Acta Med. 2013;56:57-58.

4. Camarasa A, Chiner E, Sancho J. "Nariz de payaso" como manifestación inicial de un carcinoma epidermoide de pulmón. Arch Bronconeumol. 2009;45:60-63.
} 
5. Fernández M, Parra V, Avilés J, Suárez R. Metástasis cutáneas de origen visceral. Actas Dermosifiliogr. 2013;104:841-853.

6. Alcaraz I, Cerroni L, Rutten A, Kutzner H, Requena L. Cutaneous metastases from internal malignancies: a clinicopathologic and immunohistochemical review. Am J Dermatopathol. 2012;34:347-393.

7. Marcoval J, Martínez L, Bonfill M, Valentí F. "Clown nose" as first manifestation of squamous cell carcinoma of the lung. J Dermatol Case Rep. 2017;11:9-11.

8. Chernoff K, Marghoob A, Lacouture M, Deng L, Busam K, Myskowski P. Dermoscopic findings in cutaneous metastases. JAMA Dermatol. 2014;150:429-433.

9. García I, Grasa M, Carapeto FJ. Metástasis cutánea como prime hallazgo de carcinoma pulmonar. Med Cutan Ibero Lat Am. 2009; 37:102-104

10. Abdeen Y, Amireh S, Patel A, Al-Halawani M, Shaaban H, Miller R. Cutaneous metastasis as a first presentation for lung adenocarcinoma N Am J Med Sci. 2016;8:222-225.

11. Wong C, Helm M, Kalb R, Helm T, Zeitouni N. The presentation, pathology, and current management strategies of cutaneous metastasis. N Am J Med Sci. 2013;5:499-504.

12. Nashan D, Meiss F, Braun-Falco M, Reichenberger S. Cutaneous metastases from internal malignancies. Dermatol Ther. 2010;23: 567-580.
13. Nashan D, Müller L, Braun-Falco M, Reichenberger S, Szeimies R, Bruckner-Tuderman L. Cutaneous metastases of visceral tumours: a review. J Cancer Res Clin Oncol. 2009;135:1-14

14. Sariya D, Ruth $K$, Adams-McDonnell $R$, Cusack $C$, Xu X, Elenitsas $R$ et al. Clinicopathologic correlation of cutaneous metastases. Arch Dermatol. 2007;143:613-620.

15. Nibhoria S, Kaur K, Kaur M, Kumar S. A clinicopathological and immunohistochemical correlation in cutaneous metastases from internal malignancies: a five-year study. J Skin Cancer. 2014;2014:1-5.

16. Hussein M. Skin metastasis: a pathologist's perspective. J Cutan Pathol. 2010;37:1-20.

17. Mandava A, Rao P, Konathan R. High-resolution ultrasound imaging of cutaneous lesions. Indian J Radiol Imaging. 2013;23:269-277.

18. Chun S, Kim Y, Lee J, Kim S, Lee S, Won Y, et al. Nasal tip cutaneous metastases secondary to lung carcinoma: three case reports and a review of the literature. Acta Derm Venereol. 2013;93:569-572.

19. Batson $O$. The function of the vertebral veins and their role in the spread of metastases. Ann Surg. 1940;112:138-149.

20. Wong M. An unusual skin metastasis: esophageal squamos cell carcinoma metastasizing to the nose folling nasogastric tube suture. Plast Reconstr Surg. 2005; 116:683-685.

21. Aneiros J, Arias S, García C, O’Valle F. Disfiguring basal cell carcinoma of the nose ("clown nose"). Ear, Nose Throat J. 2012;91:26-27. 